\title{
Crystal structure of diazabicyclo[2.2.2]octanium-tri(thiocyanato)zinc(II) hydrate, $\left(\mathrm{C}_{6} \mathrm{H}_{13} \mathrm{~N}_{2}\right)(\mathrm{SCN})_{3} \mathrm{Zn}\left(\mathrm{H}_{2} \mathrm{O}\right)$
}

\section{J. Pickardt and D. Röleke}

Technische Universität Berlin, Institut für Anorganische und Analytische Chemie, Straße des 17. Juni 135. D-10623 Berlin, Germany

Received July 25. 1996, CSD-No. 402563

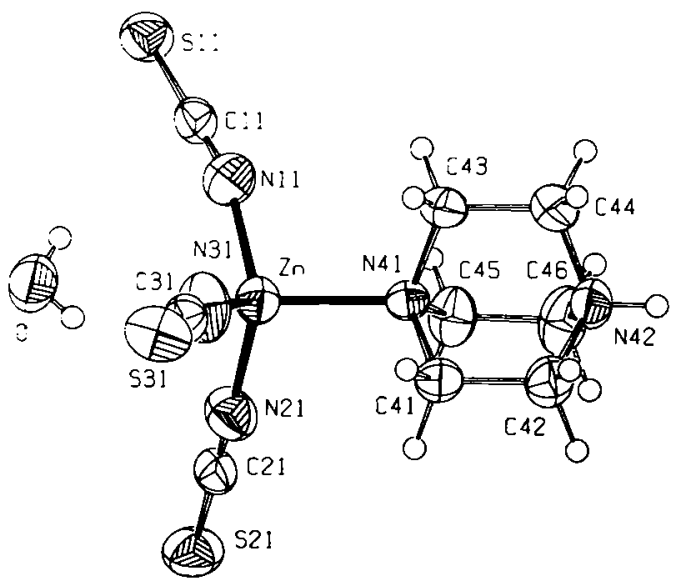

Source of material: Crystallization from an aqueous solution of zinc sulfate heptahydrate $(c=0.05 \mathrm{~mol} / \mathrm{l})$, potassium thiocyanate $(c=0.1 \mathrm{~mol} / /)$, and diazabicyclo[2.2.2]octane $(c=0.05 \mathrm{~mol} / \mathrm{l})$. $H$ positions at $C$ atoms were calculated assuming $s p^{2}$ hybridization and $\mathrm{C}-\mathrm{H}$ distances $1.08 \AA$. $\mathrm{H}$ atoms at $\mathrm{N} 42$ of the diazabicyclooctane molecule and of the water molecule refined, all thermal parameters of $\mathrm{H}$ atoms fixed.

$\mathrm{C}_{9} \mathrm{H}_{15 \mathrm{~N}} \mathrm{OSS}_{3} \mathrm{Zn}$, monoclinic, $P 12_{1} / c 1$ (No. 14), $a=7.886(2) \AA$, $b=13.015(2) \AA, c=15.636(3) \AA, \beta=98.59(2)^{\circ}, V=1586.9 \AA^{3}$, $Z=4, R(F)=0.038, R_{\mathrm{W}}(F)=0.039$.

\section{Reference}

1. Sheldrick, G. M.: SHELXI-76, Programs for Crystal Structures Determination. University of Cambridge, UK 1976.

Table 1. Parameters used for the $\mathrm{X}$-ray data collection

Crystal:

Wavelength:

$\mu$ :

Diffractometer:

Scan mode:

T measurement:

$2 \theta_{\max }:$

$\mathbf{N}(h k)_{\text {unique: }}$

Criterion for $F_{0}$

$\mathrm{N}($ param) refined.

Program:

\author{
colorless prism, size $0.5 \times 0.4 \times 0.35 \mathrm{~mm}$ \\ Mo $K_{\alpha}$ radiation $(0.71073 \AA)$ \\ $19.60 \mathrm{~cm}^{-1}$ \\ Siemens-Nicolet P21 \\ $\omega$ \\ $293 \mathrm{~K}$ \\ $55^{\circ}$ \\ 3467 \\ $F_{0}>4 \sigma\left(F_{0}\right)$ \\ 181 \\ SHELX-76
}

Table 2. Final atomic coordinates and displacement parameters (in $\AA^{2}$ )

\begin{tabular}{|c|c|c|c|c|c|}
\hline Atom & Site & $x$ & $y$ & $z$ & $\boldsymbol{U}_{\text {iso }}$ \\
\hline$H(411)$ & $4 e$ & $0.5144(4)$ & $0.0723(2)$ & $0.8863(2)$ & 0.08 \\
\hline H(412) & $4 e$ & $0.6949(4)$ & $0.0367(2)$ & $0.8387(2)$ & 0.08 \\
\hline$H(421)$ & $4 e$ & $0.8671(4)$ & $0.0940(2)$ & $0.9560(2)$ & 0.08 \\
\hline$H(422)$ & $4 e$ & $0.6867(4)$ & $0.1322(2)$ & $1.0026(2)$ & 0.08 \\
\hline$H(431)$ & $4 e$ & $0.4801(4)$ & $0.3304(2)$ & $0.7960(2)$ & 0.08 \\
\hline$H(432)$ & $4 e$ & $0.3949(4)$ & $0.2417(2)$ & $0.8635(2)$ & 0.08 \\
\hline$H(451)$ & $4 e$ & $0.8172(4)$ & $0.1556(2)$ & $0.7470(2)$ & 0.08 \\
\hline$H(452)$ & $4 e$ & $0.7319(4)$ & $0.2804(2)$ & $0.7292(2)$ & 0.08 \\
\hline$H(461)$ & $4 e$ & $0.9115(4)$ & $0.3360(3)$ & $0.8449(2)$ & 0.08 \\
\hline H(462) & $4 e$ & $0.9947(4)$ & $0.2109(3)$ & $0.8635(2)$ & 0.08 \\
\hline$H(441)$ & $4 e$ & $0.5704(5)$ & $0.3046(3)$ & $0.9779(2)$ & 0.08 \\
\hline H(442) & $4 e$ & $0.6605(5)$ & $0.3904(3)$ & $0.9098(2)$ & 0.08 \\
\hline H(1) & $4 e$ & $0.860(5)$ & $0.269(3)$ & $0.981(2)$ & 0.08 \\
\hline$H(2)$ & $4 e$ & $1.032(5)$ & $0.198(3)$ & $0.615(3)$ & 0.08 \\
\hline H(3) & $4 e$ & $0.963(6)$ & $0.124(3)$ & $0.592(3)$ & 0.08 \\
\hline
\end{tabular}

Table 3. Final atomic coordinates and displacement parameters (in $\AA^{2}$ )

\begin{tabular}{|c|c|c|c|c|c|c|c|c|c|c|}
\hline Atom & Site & $x$ & $y$ & $z$ & $U_{11}$ & $U_{22}$ & $U_{33}$ & $U_{12}$ & $U_{13}$ & $U_{23}$ \\
\hline $\mathrm{Zn}$ & $4 e$ & $0.43473(4)$ & $0.12690(2)$ & $0.69407(2)$ & $0.0398(2)$ & $0.0449(2)$ & $0.0420(2)$ & $-0.0025(1)$ & $0.0036(1)$ & $-0.0022(1)$ \\
\hline$S(11)$ & $4 e$ & $0.2547(1)$ & $0.38568(6)$ & $0.49577(5)$ & $0.0661(5)$ & $0.0460(4)$ & $0.0490(4)$ & $0.0030(3)$ & $0.0079(4)$ & $0.0044(3)$ \\
\hline$S(21)$ & $4 e$ & $0.8028(1)$ & $-0.05823(7)$ & $0.54383(6)$ & $0.0695(6)$ & $0.0672(5)$ & $0.0627(5)$ & $0.0210(4)$ & $0.0156(4)$ & $-0.0054(4)$ \\
\hline$C(11)$ & $4 e$ & $0.3192(4)$ & $0.3060(2)$ & $0.5729(2)$ & $0.044(1)$ & $0.045(1)$ & $0.042(1)$ & $0.002(1)$ & $0.004(1)$ & $-0.011(1)$ \\
\hline $\mathrm{N}(11)$ & $4 e$ & $0.3617(4)$ & $0.2491(2)$ & $0.6279(2)$ & $0.080(2)$ & $0.057(1)$ & $0.051(1)$ & $0.008(1)$ & $-0.007(1)$ & $0.003(1)$ \\
\hline$C(21)$ & $4 e$ & $0.6746(4)$ & $-0.0025(2)$ & $0.6003(2)$ & $0.047(2)$ & $0.036(1)$ & $0.045(1)$ & $-0.005(1)$ & $-0.001(1)$ & $-0.002(1)$ \\
\hline $\mathbf{N}(21)$ & $4 e$ & $0.5817(3)$ & $0.0371(2)$ & $0.6405(2)$ & $0.054(1)$ & $0.057(1)$ & $0.061(2)$ & $-0.002(1)$ & $0.013(1)$ & $-0.012(1)$ \\
\hline$S(31)$ & $4 e$ & $0.0221(1)$ & $-0.05338(7)$ & $0.81902(6)$ & $0.0712(6)$ & $0.0580(5)$ & $0.0748(6)$ & $-0.0002(4)$ & $0.0338(5)$ & $0.0037(4)$ \\
\hline $\mathrm{N}(41)$ & $4 e$ & $0.5935(2)$ & $0.1830(2)$ & $0.8016(1)$ & $0.035(1)$ & $0.032(1)$ & $0.035(1)$ & $0.0011(8)$ & $0.0060(9)$ & $0.0023(8)$ \\
\hline$C(41)$ & $4 e$ & $0.6327(4)$ & $0.0993(2)$ & $0.8671(2)$ & $0.048(2)$ & $0.035(1)$ & $0.043(1)$ & $-0.001(1)$ & $0.004(1)$ & $0.008(1)$ \\
\hline$C(42)$ & $4 e$ & $0.7503(4)$ & $0.1386(2)$ & $0.9462(2)$ & $0.062(2)$ & $0.044(1)$ & $0.046(2)$ & $-0.006(1)$ & $-0.005(1)$ & $0.011(1)$ \\
\hline$C(43)$ & $4 e$ & $0.5110(4)$ & $0.2694(2)$ & $0.8426(2)$ & $0.063(2)$ & $0.042(1)$ & $0.050(2)$ & $0.016(1)$ & $-0.004(1)$ & $-0.009(1)$ \\
\hline$C(45)$ & $4 e$ & $0.7575(4)$ & $0.2189(2)$ & $0.7758(2)$ & $0.046(2)$ & $0.061(2)$ & $0.049(2)$ & $-0.018(1)$ & $0.006(1)$ & $0.005(1)$ \\
\hline$C(46)$ & $4 e$ & $0.8792(4)$ & $0.2566(3)$ & $0.8545(2)$ & $0.057(2)$ & $0.079(2)$ & $0.059(2)$ & $-0.026(2)$ & $-0.004(2)$ & $0.013(2)$ \\
\hline$C(44)$ & $4 e$ & $0.6309(5)$ & $0.3108(3)$ & $0.9205(2)$ & $0.084(2)$ & $0.058(2)$ & $0.062(2)$ & $0.025(2)$ & $-0.018(2)$ & $-0.023(2)$ \\
\hline$C(31)$ & $4 e$ & $0.1538(4)$ & $0.0095(2)$ & $0.7705(2)$ & $0.044(2)$ & $0.053(2)$ & $0.044(2)$ & $0.003(1)$ & $0.003(1)$ & $-0.009(1)$ \\
\hline $\mathrm{N}(31)$ & $4 e$ & $0.2480(4)$ & $0.0545(2)$ & $0.7347(2)$ & $0.055(2)$ & $0.087(2)$ & $0.069(2)$ & $-0.025(2)$ & $0.016(1)$ & $-0.008(2)$ \\
\hline $\mathrm{N}(42)$ & $4 e$ & $0.7921(3)$ & $0.2485(2)$ & $0.9320(2)$ & $0.060(2)$ & $0.042(1)$ & $0.045(1)$ & $-0.004(1)$ & $-0.013(1)$ & $0.001(1)$ \\
\hline 0 & $4 e$ & $0.9522(3)$ & $0.1836(2)$ & $0.5886(2)$ & $0.056(1)$ & $0.082(2)$ & $0.057(1)$ & $-0.010(1)$ & $-0.003(1)$ & $0.015(1)$ \\
\hline
\end{tabular}

